\title{
Editorial: Recent Advances in Heterogeneous Networking for Quality, Reliability, Security and Robustness
}

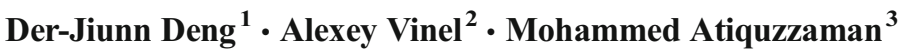

Published online: 4 February 2017

(C) Springer Science+Business Media New York 2017

\section{Editorial:}

Computer networking has been embracing increased heterogeneity since its inception, in terms of the range of the applications that it has to support, various communication technologies that it can run on, and hierarchical, hybrid and heterogeneous techniques that it has to rely on to meet the challenges from both the diverse application requirements and communication technologies.

The goal of this special issue is to publish both state-of-theart and prospective papers that present advances towards the performance enhancement of heterogeneous networking, particularly for quality, experience, reliability, security and robustness to heterogeneous networking and infrastructure for the future networks. The call for papers for this special issue attracted submissions from Asia, Africa, Europe, and the US covering a wide range of topics in the related field. Each paper was carefully evaluated by at least three reviewers. This careful evaluation process has allowed us to select 12 high quality research papers. We strongly believe that the selected papers

Der-Jiunn Deng

djdeng@cc.ncue.edu.tw

Alexey Vinel

alexey.vinel@gmail.com

Mohammed Atiquzzaman

atiq@ou.edu

1 Department of Computer Science and Information Engineering, National Changhua University of Education, No. 2, Shi-Da Rd., Changhua 500, Taiwan

2 School of Information Technology, Halmstad University, Kristian IV:s väg 3, Halmstad 301 18, Sweden

3 Department of Computer Science, The University of Oklahoma, 110 W. Boyd St, Norman 73019, OK, USA will make a significant contribution to this field. We present a brief overview of each manuscript in the following.

The dynamics of cloud resources determined that the traditional static evaluation indicators cannot accurately describe resource states. In the paper entitled "Dynamically Weighted Load Evaluation Method Based on Self-adaptive Threshold in Cloud Computing" authored by Liyun Zuo, et al. proposes a Self-adaptive threshold based Dynamically Weighted load evaluation Method (termed SDWM) to evaluate the load state of the resource through a dynamically weighted evaluation method.

The second paper, "A Channel Bonding based QoS-aware OFDMA MAC Protocol for the Next Generation WLAN", authored by $\mathrm{Hu}$ Zhou, et al., proposes a Channel Bonding based QoS-aware Orthogonal Frequency Division Multiple Access (OFDMA) MAC protocol (CBQO) to achieve high throughput by utilizing the wide bandwidth while decreasing the collision probability among stations in IEEE 802.11ax WLANs.

The third paper, "Hierarchical Packet Pre-dropping Approach for Improved MPEG-4 Video Transmission in Heavily Loaded Wireless Networks" by Chih-Heng Ke, et al. proposes a hierarchical packet pre-dropping (HPPD) approach to improve the MPEG-4 video transmission quality in heavily loaded IEEE 802.11e WLANs.

The fourth paper, "Design and Implementation of Various File Deduplication Schemes on Storage Devices", by Kuan$\mathrm{Wu} \mathrm{Su}$, et al. proposes several file deduplication schemes built in the private cloud storage appliance to increase the space efficiency in private cloud storage systems as well as preserve network bandwidth.

The fifth paper, "An Effective Failure Recovery Mechanism for SIP/IMS Services", by Whai-En Chen, et al. investigates the performance of different database (DB) access mechanisms in $4 \mathrm{G}$ networks, and then proposes a loss- 
recovery enhancement for the Write-Back (WB) mechanism to improve the successful call setup probability.

The sixth paper, "The Quick Radio Fingerprint Collection Method for a WiFi-Based Indoor Positioning System", by Hung-Huan Liu proposes a quick radio fingerprint collection (QRFC) algorithm to collect the sampling information for a WiFi-Based Indoor Positioning System.

The seventh paper, "A New QoE-Driven Video Cache Management Scheme with Wireless Cloud Computing in Cellular Networks", by Yumei Wang, et al. proposes a new QoE-driven video cache management scheme with the consideration of the parameters from client, base station, and RAN cache server for video provisioning, with statistics of video popularities and under limited cache capacity.

The eighth paper, "A CSMA/CA-based Media Access Protocol Using the Registered Backoff Time Vector (RBTv) Mechanism for M2M Communications", by Rung-Shiang Cheng, et al. proposes an enhanced IEEE 802.11 CSMA/CA media access scheme, CSMA/CARBTv, to improve the overall transmission delay and the average throughput for Machine-to-Machine (M2 M) communication.

The ninth paper, "Fast-start Video Delivery in Future Internet Architectures with Intra-domain Caching", by Zhi Liu, et al. proposes to encode the video content using SVC for the fast-start video delivery in $\mathrm{CCN}$.

The tenth paper, "Differentiating and Scheduling LTE Uplink Traffic Based on Exponentially Weighted Moving Average of Data Rate", authored by Fang-Chang Kuo, et al., develops an algorithm using exponentially weighted moving average (EWMA) to define the measurement of GBR, and then present a scheduling scheme referred to as Allocate As Granted-version 2 (AAG-2) to ensure a minimum QoS for all in-progress GBR sessions.

In the next paper entitled "On Different-Dimensional Deployment Problems of Hybrid VANET-Sensor Networks with QoS Considerations", authored by Chun-Cheng Lin, et al., investigates different-dimensional deployment problems in HVSNs, with objective to minimize the total distance and the total number of hops from sensors to the closest RSU, under constraints of the maximal number of hops and capacity.

The last article titled "Performance Analysis of the Industrial Wireless Networks Standard: WIA-PA", authored by Meng Zheng, et al., analyzes salient features of Industrial Automation-Process Automation (WIA-PA): mesh-star architecture, two-level aggregation, and adaptive frequency hopping, whose working principles are explicitly illustrated in comparison to the other two mainstream industrial wireless networks standards: WirelessHART and ISA 100.11a.
Acknowledgements As the guest editors of this special issue, we would like to thank all authors who have submitted papers to the special issue and in particular those whose papers have been accepted for this special issue. Assistance from the editorial staff of the Mobile Networks and Applications is also much appreciated. Besides, the guest editors wish to acknowledge all those who have generously given their time to review the papers submitted for consideration for inclusion in this special issue. Finally, our special thanks go to Dr. Imrich Chlamtac (editor-in-chief) for his valuable support throughout the preparation of this special issue.

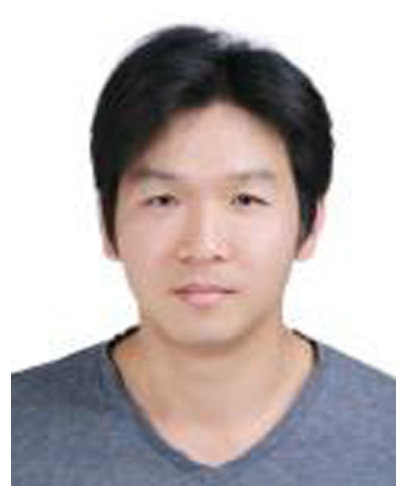

Der-Jiunn Deng (M'10) received the Ph.D. degree in electrical engineering from the National Taiwan University in 2005. He joined the National Changhua University of Education as an Assistant Professor in the Department of Computer Science and Information Engineering in August 2005 and then became a Distinguished Professor in August 2016. In 2010, 2011, and 2012, he received the Research Excellency Award of National Changhua University of Education. In 2012 and 2015, he also received the Outstanding Faculty Research Award of National Changhua University of Education. His research interests include multimedia communication, quality-of-service, and wireless networks. Dr. Deng served or is serving as an editor and guest editor for several technical journals. He also served or is serving on several symposium chairs and technical program committees for IEEE and other international conferences. Dr. Deng is a member of the IEEE.

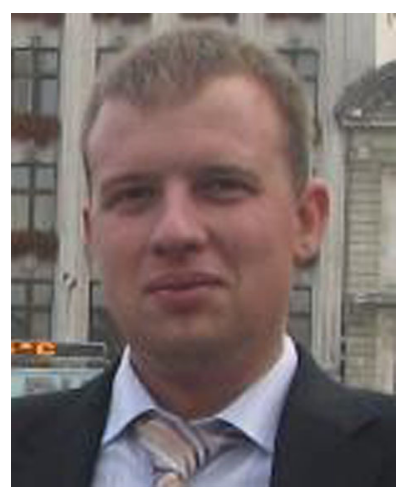

Alexey Vinel [M'07, SM'12] is a professor of data communications with the School of Information Technology, Halmstad University, Sweden. He received his Bachelors (Hons.) and Masters (Hons.) degrees in information systems from SaintPetersburg State University of Aerospace Instrumentation, Russia, in 2003 and 2005, respectively, and the Ph.D. degrees in technology from the Institute for Information Transmission Problems, Russia in 2007, and Tampere University of Technology, Finland in 2013. He is currently involved in research projects on vehicular networking standards, advanced drivers' assistance systems, and autonomous driving. He has been an associate editor for IEEE Communications Letters since 2012. 


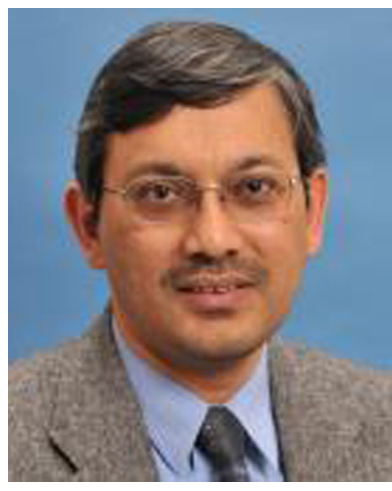

Mohammed Atiquzzaman is a professor of Computer Science at The University of Oklahoma. He teach courses in Data Networks and Computer Architecture. His research interests and publications are in next generation computer networks, wireless and mobile networks, satellite networks, switching and routing, optical communications and multimedia over networks. Many of the current research activities are supported by the National Science Foundation (NSF), National Aeronautics and Space Administration (NASA) and the U.S. Air Force. Prof. Mohammed Atiquzzaman serves as the Editor-in-Chief of Journal of Network and Computer Applications, Editor-in-Chief of the Vehicular Communications journal and associate editor of IEEE Communications Magazine, Journal of Wireless and Optical Communications, International Journal of Communication Systems, International Journal of Sensor Networks, International Journal of Communication Networks and Distributed Systems, and Journal of Real-Time Image Processing. 